\title{
Cryo-Recanalization of Huge Endobronchial Pulmonary Adenocarcinoma
}

\section{Falkenstern-Ge RF ${ }^{1 *}$, Kimmich $\mathbf{M}^{1}$, Wohlleber $\mathbf{M}^{1}$, Friedel $\mathbf{G}^{2}$, Biwer AS ${ }^{3}$ and Alscher $\mathbf{M}^{4}$}

${ }^{1}$ Division of Pulmonology, Center for Pulmonology and Thoracic Surgery, Teaching Hospital of the University of Tuebingen, Germany

${ }^{2}$ Division of Thoracic Surgery, Director of Center for Pulmonology and Thoracic Surgery, Teaching Hospital of the University of Tuebingen, Germany

${ }^{3}$ Department of Clinical Pathology, Robert Bosch Central Hospital, Teaching Hospital of the University of Tuebingen, Germany

${ }^{4}$ Department of General Internal Medicine and Nephrology, Chief Medical Director of Robert Bosch Hospital, Teaching Hospital of the University of Tuebingen, Germany

\begin{abstract}
We were able to recanalize the major lumen of the right main bronchial branch, which was completely blocked because of huge tumor mass from the right upper lobe. With the cryo-therapy, we could freeze and remove the major portion of this huge tumor mass.
\end{abstract}

Keywords: Adenocarcinoma; Lung cancer; Bronchoscopy; Intervention

Abbreviations: EGFR: Epidermal Growth Factor Receptor; ALK: Anaplastic Lymphoma Kinase; ROS-1: Proto-Oncogene TyrosineProtein Kinase ROS; PDL1: Programmed Death Ligand-1

\section{Case Report}

A 70-years-old strong smoker (50 pack years) was referred to our center due to increasing dyspnea, coughing and hemoptysis. The patient was in reduced general clinical conditions.

The contrast-enhanced tomography revealed huge tumor mass of the right bronchial system (Figure 1). The staging procedure showed multiple osseous, hepatic and lymphatical metastasis. There were no surgical options; a systemic palliative therapy was to be initiated after the histological and immunhistological examination.

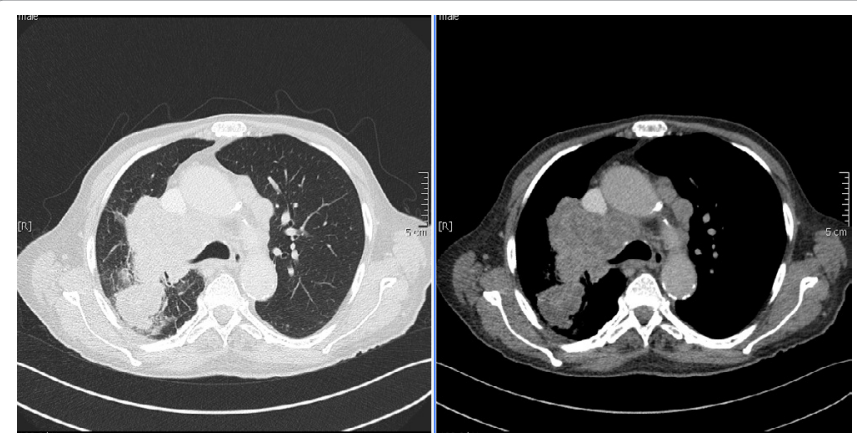

Figure 1: Huge tumor mass of the right bronchial- tree, upper lobe, middle lobe as well as lower lobe were partially and almost completely blocked through the tumor.

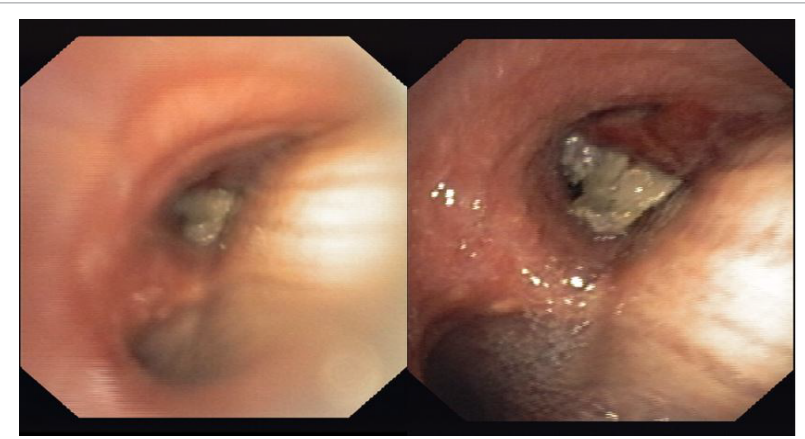

Figure 2: Endobronchial finding during rigid bronchoscopy showed huge tumor mass narrows the right bronchial main stem. Only a slit opening was visible.

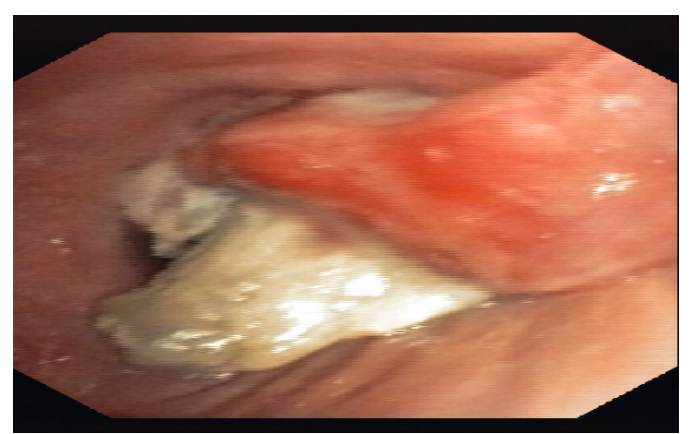

Figure 3: A closer look at the huge endobronchial tumor mass from Figure 2.

We carried out rigid bronchoscopy. Endobronchial we confirmed a huge tumor mass, which infiltrated and blocked the entrance of the entire right main branch of bronchial system (Figures 2 and 3). After the initial preparation with instillation of iced $\mathrm{NaCl} 0.9 \%$ and Xylometazoline, we could freeze and remove the major exophytic portions of the tumor mass with cryo-probe (Figures $4 \mathrm{a}$ and $4 \mathrm{~b}$ ). The origin of the tumor was most likely to be located at segment 1 of right upper lobe. Mild bleeding problems could be solved with topical application of xylometazoline. After the successful de-bulking, the bronchial ostia of middle lobe and lower lobe were clear visible, with no signs of exophytic tumor tissues.

The patient recovered well from this procedure, also he reported to breath a lot easier after the endobronchial tumor de-bulking.

Histopathological examination of the tumor biopsy revealed strands of tumor cells infiltrating desmoplastic stroma. The main architectural pattern was papillary, with enlarged moderately pleomorphic tumor cells with hyperchromatic nuclei resting on fibrovascular cores. Immunohistochemistry showed reactivity for CK7 without expression of TTF-1 or p40. In conclusion, a diagnosis of papillary adenocarcinoma

*Corresponding author: Falkenstern-Ge RF, Division of Pulmonology, Klinik Schillerhoehe, Center for Pulmonology and Thoracic Surgery, Teaching Hospital of the University of Tuebingen, Solitude Str. 18, 70839 Stuttgart-Gerlingen, Germany, Tel: 07156-2030; E-mail: Roger-Fei.Falkenstern-Ge@rbk.de

Received: April 26, 2017; Accepted: May 13, 2017; Published: May 16, 2017

Citation: Falkenstern-Ge RF, Kimmich M, Wohlleber M, Friedel G, Biwer AS, et al. (2017) Cryo-Recanalization of Huge Endobronchial Pulmonary Adenocarcinoma. Oncol Cancer Case Rep 3: 129.

Copyright: (c) 2017 Falkenstern-Ge RF, et al. This is an open-access article distributed under the terms of the Creative Commons Attribution License, which permits unrestricted use, distribution, and reproduction in any medium, provided the original author and source are credited. 
Citation: Falkenstern-Ge RF, Kimmich M, Wohlleber M, Friedel G, Biwer AS, et al. (2017) Cryo-Recanalization of Huge Endobronchial Pulmonary Adenocarcinoma. Oncol Cancer Case Rep 3: 129.

of the lung was rendered. Extended tumor work-up revealed no EGFR mutations or ALK or ROS rearrangements. PDL-1 was not expressed by the tumor cells.

Therefore, a palliative system therapy with carboplatin and nabpaclitaxel was initiated. Due to chronic renal failure, systemic therapy with pemetrexed was not feasible.

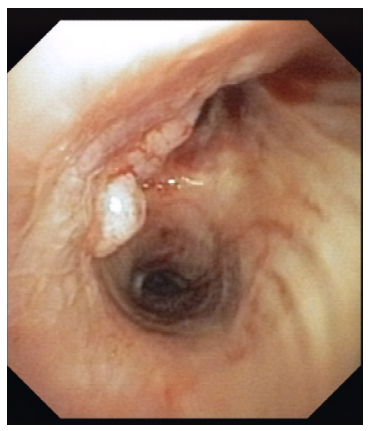

Figure 4a: Successful cryo- recanalization, the right main branch was recanalized after effective tumor de-bulking.

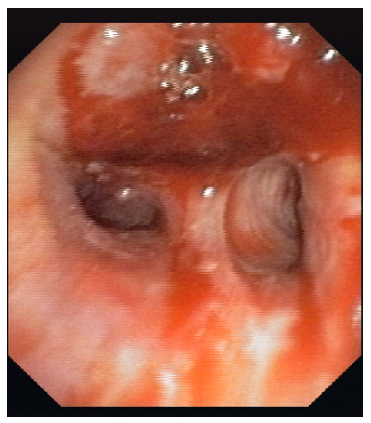

Figure 4b: The origin of the huge endobronchial tumor mass was located in the right upper lobe most likely segment 1.

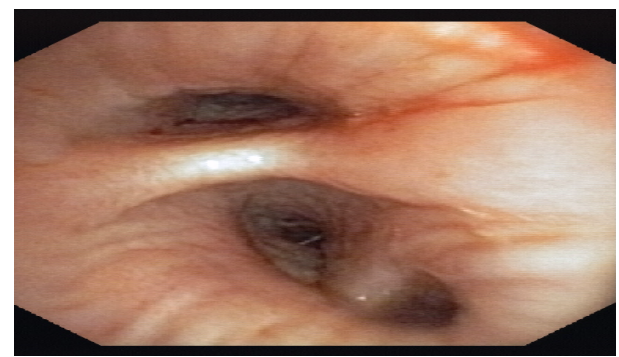

Figure 5: The middle lobe and lower lobe were also recanalized after the successful endobronchial tumor de- bulking.

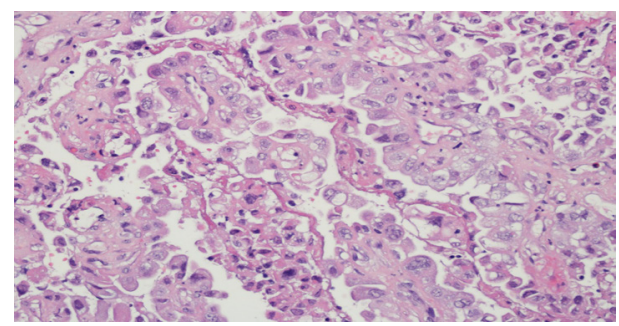

Figure 6a: Haematoxylin and eosin (H\&E)-stained section of papillary lung adenocarcinoma (20x magnification).

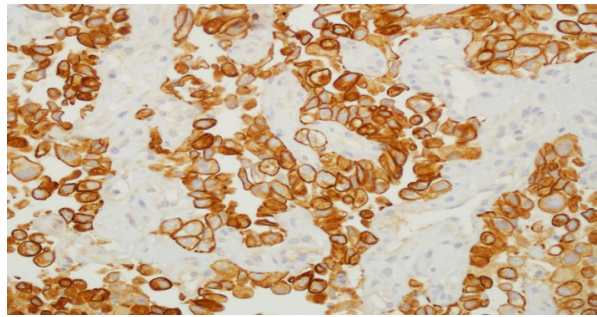

Figure 6b: The adenocarcinoma shows strong staining for CK (20x magnification).

\section{Discussion}

Huge endobronchial tumors could cause severe airway obstruction with symptoms such as dyspnea, cough, and hemoptysis.

The established technique cryo-recanalization is a therapy, in which the physician is able to freeze the endobronchial tumor mass and later removing the frozen malignant tissue. In this way, it results in a very effective rapid tumor de-bulking. Through the rapid de-bulking, the airway lumen can be recanalized [1-3].

Cryosonde biopsy and recanalization was found to be effective, this method of treatment is appropriate for use against localized disease; diffuse metastases cannot be handled with cryotherapy $[1,2]$. In our case, we were able to remove huge tumor mass by using cryosonde, in which we totally recanalized the airway from the right main bronchial branch (Figure 5).

Some other therapeutic options have rapid clinical responses (argon plasma coagulation, laser and electrocautery), but are mostly associated with significant complications, including bleeding, fire, airway perforation and re-stenosis [4].

Hetzel et al. [5] have successfully observed that the tissue samples collected during the cryo-recanalization procedure are of high quality and much larger than conventional biopsy samples [5].

Reported restenosis rates after cryotherapy range between $24 \%$ and $28 \%$ occurring within 2 months to 4 years $[2,6]$ of the procedure. Our patient had so far, no recurrence as seen on check bronchoscopy after 8 weeks. Further endobronchial checks will be performed after the first two cycles of palliative chemotherapy.

Another advantage of cryotherapy is that oxygen may not be stopped during the procedure, as is mandated during other modalities $[2,4]$. Despite its high efficacy, low cost, and relative safety, this technique remains underutilized. Cryo-recanalization is a safe, cheap and effective method for the immediate management of airway obstruction [2,4]. Large fragments of tissue could be removed with a cryoprobe, which, besides achieving an excellent recanalization, also give a better chance to obtain a proper histopathological diagnosis [5] (Figures 6a and 6b).

Hetzel et al. [2] made a prospective study referring to flexible bronchoscopy used for cryo-recanalization of 60 patients with highgrade stenosis of the respiratory tract from exophytic tumors. Hetzel et al. had excellent results in their study with fifty (83\%) of 60 patients were successfully or partially successfully treated. Tumor bleeding occurred in 6 patients but was stopped with argon plasma coagulator treatment in all patients not requiring rigid bronchoscopy [2].

Furthermore, in contrast to laser therapy, with its high-thermal energy release, cryo-recanalization can be performed in patients with 
Citation: Falkenstern-Ge RF, Kimmich M, Wohlleber M, Friedel G, Biwer AS, et al. (2017) Cryo-Recanalization of Huge Endobronchial Pulmonary Adenocarcinoma. Oncol Cancer Case Rep 3: 129.

coated airway stents [7]. Another advantage of cryo is that it does not damage bronchial cartilage because of its lower water content. Chawla et al. also reported a very interesting case of successful cryorenalization of endo-bronchial carcinoid tumor [8]. The most common complications of cryo-surgery are bleeding, bronchial wall damage and hypoxia [8].

\section{Conclusion}

More case reports are much needed, in order to show the therapeutic effects of this innovative method of solving endobronchial stenosis, which are caused by tumor. Based on our opinion, the therapy with cryo-recanalization should be more utilized.

\section{References}

1. Schumann C, Lepper PM, Barth TF, Möller P, Krüger S (2009) Successfu immediate cryorecanalization of a simultaneous high-grade tracheal and bronchial stenosis as rare manifestations of bronchial-associated lymphoid tissue lymphoma. J Thorac Cardiovasc Surg 137: e17-19.
2. Hetzel M, Hetzel J, Schumann C, Marx N, Babiak A (2004) Cryorecanalization: A new approach for the immediate management of acute airway obstruction. $J$ Thorac Cardiovasc Surg 127: 1427-1431.

3. Schumann C, Hetzel M, Babiak AJ, Hetzel J, Merk T, et al. (2010) Endobronchia tumor debulking with a flexible cryoprobe for immediate treatment of malignant stenosis. J Thorac Cardiovasc Surg 139: 997-1000.

4. Bolliger CT, Mathur PN, Beamis JF, Becker HD, Cavaliere S, et al. (2002) European Respiratory Society/American Thoracic Society. ERS/ATS statement on interventional pulmonology. European Respiratory Society/American Thoracic Society. Eur Respir J 19: 356-373.

5. Hetzel J, Hetzel M, Hasel C, Moeller P, Babiak A (2008) Old meets modern: The use of traditional cryoprobes in the age of molecular biology. Respiration 76: 193-197.

6. Deygas N, Froudarakis M, Ozenne G, Vergnon JM (2001) Cryotherapy in early superficial bronchogenic carcinoma. Chest 120: 26-31.

7. Dumon JF, Reboud E, Garbe L, Aucomte F, Meric B (1982) Treatment of tracheobronchial lesions by laser photoresection. Chest 81: 278-284.

8. Chawla RK, Madan A, Chawla A, Arora HN (2015) Cryo- recanalization in a case of carcinoid tumor- An interesting case report. Lung India 32: 511-514. 